\title{
The relationship of the tertiary lymphoid structures with the tumor-infiltrating lymphocytes and its prognostic value in gastric cancer
}

Nana Zhang, ${ }^{1,2,3}$, Guanjun Zhang ${ }^{3,4}$, Depu Wang ${ }^{3,5}$, Hao Liu ${ }^{1,2,3}$, Yuchi Zhang ${ }^{1,2}$, Vivian Adiila Ayarick ${ }^{3}$, Xuan Han', ${ }^{1,2}$ Yi Lv'1,2, Yili Wang ${ }^{1,3}$

\begin{abstract}
${ }^{1}$ Center for Regenerative and Reconstructive Medicine, Med-X Institute of Western China Science \& Technology Innovation Harbour, The First Affiliated Hospital of Xian JiaoTong University, China

${ }^{2}$ National Local Joint Engineering Research Center for Precision Surgery \& Regenerative Medicine, The First Affiliated Hospital of Xi'an Jiaotong University, China

${ }^{3}$ Institute for Cancer Research School of Basic Medical Science of Xi'an Jiaotong University, China

${ }^{4}$ Department of Pathology, The First Affiliated Hospital of Xi'an Jiaotong University 710061, China

${ }^{5}$ Department of Science and Technology, The First Affiliated Hospital of Xi'an Jiaotong University, China
\end{abstract}

Submitted: 24 October 2020; Accepted: 30 July 2021

Online publication: 2 August 2021

Arch Med Sci

DOI: https://doi.org/10.5114/aoms/140622

Copyright (c) 2022 Termedia \& Banach

\section{Abstract}

Introduction: To explore the relationship between the tertiary lymphoid structures (TLSs) and tumor-infiltrating lymphocytes (TILS), and their distribution characteristics as well as the prognostic value in gastric cancer (GC). Material and methods: The TLSs and four subtypes of TILs were assessed by immunohistochemical (IHC) staining. The presence of MECA-79 positive high endothelial venules (HEVs) identified among the ectopic lymphocyte aggregation area in the GC tissue was defined as valid TLSs. The number of labeled TILs was observed in 5 fields of the most positive cells in the tumor center, invasive edge and within the TLSs, at a field of vision $\times 40$.

Results: The TLS distribution was significantly higher in the tumor invasive edge than the tumor center $(p<0.001)$. Similarly, the infiltrating density of CD8+T cells and GrB+T cells was statistically significantly higher in the tumor infiltrating edge than the tumor center. The total number of TILs and FOXP3+T cells showed a contrary distribution. There was a positive correlation of the density of TLSs and TILs with both the location and the immune phenotype. A higher frequency of TILs and TLSs is often associated with favorable clinicopathologic parameters. Higher numbers of peri-TLSs $(p=0.007)$, peri-CD $8+(p=0.019)$ and peri-GrB+TILs $(p=0.032)$ were significantly correlated with the favorable overall survival. Multivariate analysis revealed that the densities of TILs $(p=0.019)$ and TLSs $(p=0.037)$ were independent prognostic predictor for GC patients.

Conclusions: We provide evidence that TLSs were positively associated with lymphocyte infiltration in GC. Thus, the formation of TLSs predicts advantageous immune system function and can be considered as a novel biomarker to stratify the overall survival risk of untreated GC patients.

Key words: tertiary lymphoid structures, tumor-infiltrating lymphocytes, gastric cancer, prognosis.

\author{
Corresponding author: \\ Yi LV \\ Center for Regenerative \\ and Reconstructive Medicine \\ Med-X Institute of Western \\ China Science \& Technology \\ Innovation Harbour \\ National Local Joint \\ Engineering Research Center \\ for Precision Surgery \\ \& Regenerative Medicine \\ The First Afliated Hospital \\ of Xi'an Jiaotong University \\ China \\ E-mail: luyi169@126.com \\ Yili Wang \\ Center for Regenerative \\ and Reconstructive Medicine \\ Med-X Institute of Western \\ China Science \& Technology \\ Innovation Harbour \\ The First Affiliated Hospital \\ of Xian JiaoTong University \\ Institute for Cancer Research \\ School of Basic Medical \\ Science of Xi'an Jiaotong \\ University \\ China \\ E-mail: \\ wangyili@mail.xjtu.edu.cn
}




\section{Introduction}

The tumor microenvironment (TME) has been widely implicated in tumorigenesis. It harbors many components that interact with tumor cells through the circulatory and lymphatic systems to influence the development and progression of cancer [1]. The immune cells are important constituents of the tumor stroma and critically take part in carcinogenesis in elimination, equilibrium and escape of cancer immunoediting processes [2]. Growing evidence suggests that the cross-talk between cancer cells and immune cells ultimately results in an environment that fosters or inhabits tumor growth and metastasis[3]. Therefore, the examination of the immune components of TME is essential for evaluations of cancer prognosis and treatment response.

Tertiary lymphoid structures (TLSs) are ectopically aggregated nascent lymphoid structures within tissues in non-lymphoid organs and characterized by high endothelial venules (HEVs) [4]. The structure and function of the TLSs are highly similar to those of secondary lymphoid organs (SLOs) such as segregated T and B cell zones, presence of follicular dendritic cell networks, HEVs, and specialized lymphoid fibroblasts and have mechanisms to support local adaptive immune responses toward locally expressed antigens [5]. Recently, the presence of TLSs has been identified in many solid organ tumors, and it was reported to be closely related to the long-term survival of cancer patients $[6,7]$. Current studies indicated that TLSs can improve the immunotherapy effect for cancer patients $[8,9]$. A successful anti-tumor immune response is always controlled by a complex series of interactions between tumor cells and immune cells $[10,11]$. The TLSs in the TME are composed of a T cell-rich zone with mature dendritic cells (DCs) juxtaposing a B cell follicle with germinal center characteristics [12]. Therefore, the TLSs are involved in the presentation of neighboring tumor antigens to $T$ cells by DCS and activation, proliferation and differentiation of $T$ and $B$ cells within the TME [13-15], which greatly affect tumor development and progression [16].

Tumor-infiltrating lymphocytes (TILs) are the major effectors encountering malignancy in the frontier of the TME $[17,18]$. It is clear that the TILS are closely involved in the formation of the local immune microenvironment, anti-tumor immune response, and immunotherapy $[19,20]$. However, TILs not only participated in the direct killing effect on tumors but also regulated the anti-tumor immune response and even impaired the anti-tumor response [21, 22]. Functional phenotypes of lymphocytes profoundly facilitate the exploration of TIL subsets in situ. Various combinations of TIL subpopulation detection panels have been reported. A study confirmed the densities of $\mathrm{CD} 3+\mathrm{T}$ cells and cytotoxic CD8+ T cells in the tumor and the invasive margin with a clear evaluation value for immunotherapy and prognosis for patients with colon cancer [23]. FOXP3+ regulatory cells (Tregs), playing a critical role in immune tolerance and deficiency of anti-tumor immunity, were often used as a negative anti-tumor parameter [24]. In this study, we therefore mainly explored the TLSs' relation with cytotoxic CD8+T cell and FOXP3+Treg supplemented with activated CTLs (GrB+T cell) and primed CD4+T cells (OX40+T cells, inducing cytokine production and maintaining the activity of $T$ helper cells).

Preclinical and clinical data support the role of TLSs and TILs in modulating the anti-tumor immune function of the host active immune system [8, 21]. Structurally, TLSs are involved in the organization of immune cells in the TME and play a similar function to lymphoid organs by recruiting naive $T$ and $B$ cells to the tumor site through the related chemokines and cytokines, and exert their corresponding immune function [25]. The TILs are often exposed in the TME to interact directly with tumor cells. However, less attention has been paid to the relationship between the two important immune components: TLSs and TILs. Moreover, the localization and relationship between TILs and TLSs in gastric cancer (GC) have never been elucidated. Understanding the location, prognostic value of TLSs and TILs, and their relationship is of great practical significance to mobilize a variety of immune functions in the body and promote the synergistic anti-tumor effect of immune cells. Thus, we performed a comprehensive histological analysis of TLSs and TILs in the surgically resected GC tissue from a cohort of 147 GC patients, which is of great importance to provide new concepts and therapeutic directions for clinical immunotherapy of GC.

\section{Material and methods}

\section{Patients and tissue specimens}

Patients who were diagnosed with gastric adenocarcinoma and underwent $\mathrm{GC}$ resection surgery between January 2001 and December 2003 in the First Affiliated Hospital of Xl'an Jiaotong University were included in this study. It was approved by the Medical Ethics Committee of the First Affiliated Hospital of Xi'an Jiaotong University.

Biopsy samples were collected from 180 patients with gastric adenocarcinoma who underwent subtotal or total gastrectomy with local lymphadenectomy at the Department of Pathology. All specimens were formalin-fixed, embedded in paraffin, cut into $4-\mu \mathrm{m}$-thick tissue sections, and stored at $-80^{\circ} \mathrm{C}$ until use. Patients were incorporated according to the following criteria: 1. Pathological diagnosis was primary gastric adenocarcinoma; 2. Without preoperative chemotherapy or radiotherapy; 3. Retained paraffin-fixed 
tissue was sufficient, and the structure was clear; 4. Cases had complete medical records and followup information. Patients were followed up for 10 years; the survival time is from the date of surgery to the end of follow-up. A total of 147 cases were included in this study. The detailed information is described in Table I of our previous study (Supplementary Table I) [26]. The survival time of patients was between 1 and 123 months, and the median survival time was 50.6 months.

\section{Immunohistochemical staining for displaying TLSs and TILs}

The tissue samples were fixed in formalin and embedded in paraffin, and the paraffin blocks were sliced into $4 \mu \mathrm{m}$ sections to conduct immunohistochemical (IHC) staining. Six serial sections of each paraffin-embedded tumor block were cut, one for $\mathrm{H} \& \mathrm{E}$ staining and five for IHC staining. The primary antibodies for TIL subsets were mouse monoclonal anti-human CD8 (DakoCytomation, Glostrup, Denmark; 1 : 100 dilution), granzyme B (Novocastra, Newcastle, UK; 1 : 100), OX40 (Novocastra; 1 : 30), FOXP3 (Abcam, Cambridge, UK; 1 : 50). The identification for the TLSs was the primary antibody to MECA-79 (1: 300 dilution, Santa Cruz, California, USA), which recognizes sulfate-dependent carbohydrate epitopes of peripheral node addressin (PNAd) expressed in endothelial cells of HEVs.
The IHC was performed as recommended by the manufacturer. The antigen retrieval was done by heat-induced epitope retrieval methods for $1 \mathrm{~min}$ and $30 \mathrm{~s}$ in citric buffer ( $\mathrm{pH}$ 6.0). The detailed steps were the same as in our previous study [26]. Dako EnVision (DakoCytomation, Denmark) was used as the secondary antibody. Negative controls were done by omitting the primary antibodies. The tonsil tissue was used as positive controls.

\section{Histological and immunohistochemical evaluation}

The H\&E-stained slides were reviewed by two pathologists (YLW and GJZ) to reconfirm the accuracy of the pathological data.

Evaluation of TLSs and TLSs classification. TLSs are characterized by B-cell follicles, T-cell zones, and specialized vessels known as HEVs. Referring to previous studies [27], the presence of MECA-79 positive HEVs identified among the ectopic lymphocyte aggregation area is defined as valid TLSs. Reference methods for the evaluation of spatial distributions of TLSs were based on a similar report on breast cancer [28]. We defined tumor-associated TLSs as either intratumoral TLSs (intra-TLSs) located within the tumor center, which is the interior of the solid tumor and enclosed by the tumor tissue in the invasive margin including the tumor parenchyma and
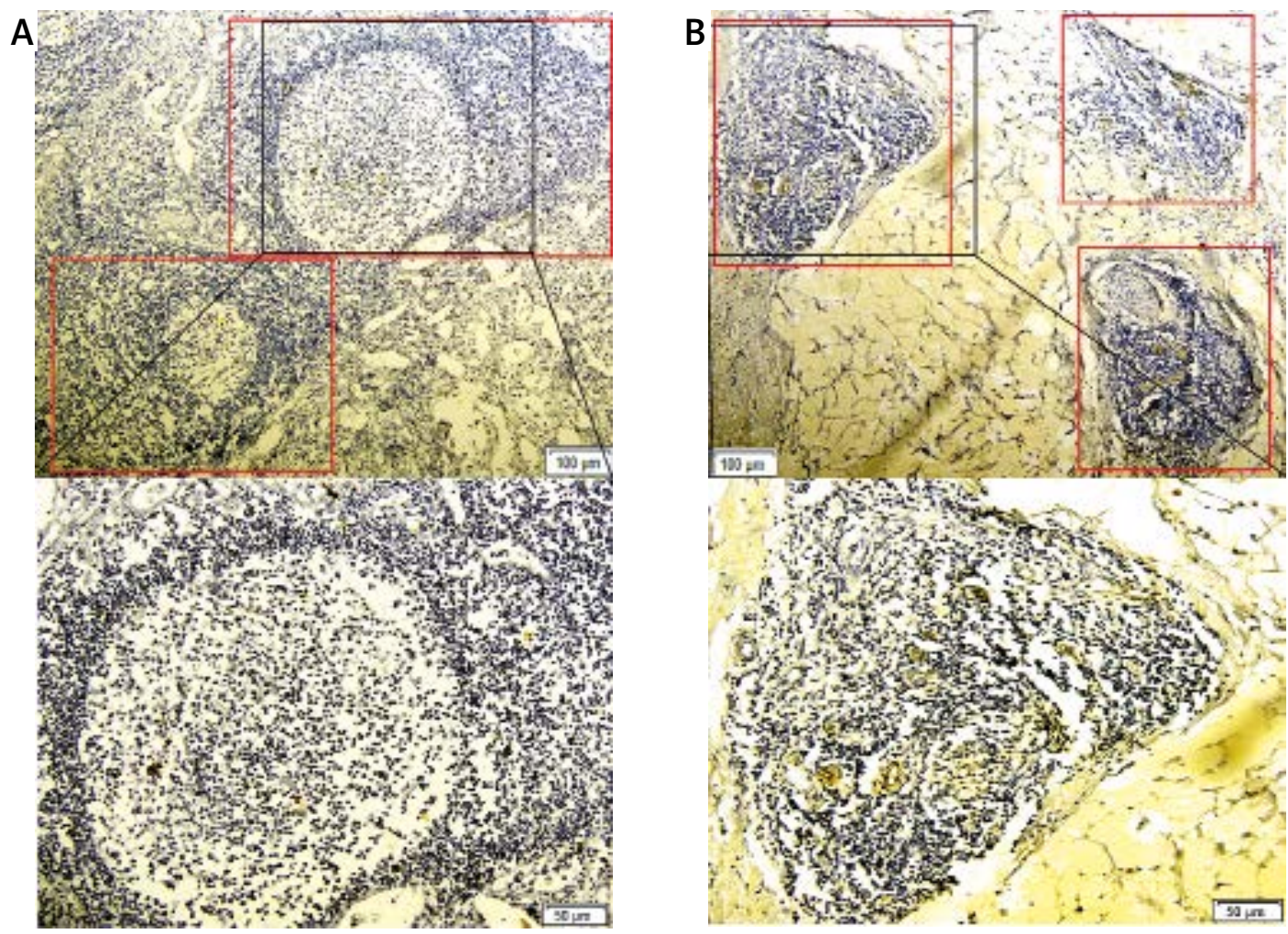

Figure 1. Intratumoral-TLSs (intra-TLSs) (A) and peritumoral-TLSs (peri-TLSs) (B) in the GC tissue, respectively. A complete TLSs structure was rolled up by a red rectangle. The picture below which it corresponds, is a local amplification of the structure of TLSs 
stroma, or peritumoral TLSs (peri-TLSs) adjacent to the invasive margin of the GC tissue (Figure 1). The total number of TLSs is the sum of intra-TLSs and peri-TLSs. After the TLSs in the whole slides were counted, the cases were divided into TLSs-high and TLSs-low groups according to the median for further analysis.

Evaluation of TILs and TILs classification. The distribution of TILs was approached in the tumor center, invasive edge, and within the TLSs, respectively. The numbers of labeled TILs were counted in 5 fields of the most positive cells by the classical counting method with the light microscope using an ocular grid at $\times 400$ magnification [29]. Then the mean value for each vision of each case was calculated to represent the TIL density for this case of this region. Counting was performed twice for each slide by two viewers (NNZ and DPW). The mean value of TILs was taken for further analysis, and the cases were divided into TILs-high and TILs-low groups according to the median for further analysis. The TILs in the two regions were also named intra-TILs and peri-TILs, respectively, and the TILs in the TLSs were termed TLSs-TILs.

\section{Statistical analysis}

The SPSS software 13.0 (Chicago, IL) was used for statistical analysis. The t-test was used to analyze the discrepancy of TILs and TLSs in intratumoral and peritumoral regions. Pearson correlation analysis was used to evaluate the relation between TLSs and TILs in a different area. The LSD t-test was used to analyze the numerical superiority of the four types of TILs in TLSs and intratumoral and peritumoral regions. The survival rates were calculated by the Kaplan-Meier method, and differences were obtained by the log-rank test. Correlations between the TLSs and the TILS and other clinicopathological variables were determined by the chi-square test. Univariate and multivariate survival analyses were carried out by Cox proportional hazards models. The R programming language was adopted for the power analysis. All the analyses were 2-sided, and $p<0.05$ was considered as statistically significant.

\section{Results}

\section{Distribution of TLSs and TILS}

Among the total 147 cases, 11 cases (7.48\%) showed no TLSs in the intratumoral or peritumoral region, and there were 17 cases (11.56\%) without peri-TLSs and 21 cases (14.29\%) without intra-TLSs. The FOXP3+ and CD8+TILs were positively distributed in the intratumoral and peritumoral regions of all the cases $\left(\chi^{2}=6.217, p=0.102\right)$. Two cases in the intratumoral and 4 cases in the peritumoral region had a negative infiltration of OX40+T cells. There were 7 cases without $\mathrm{GrB}+\mathrm{T}$ cells at the invasive margin, 5 cases without GrB+T cells in the tumor center, and a total of 5 cases with a negative infiltration of GrB+T cells.

The TLS distribution was significantly higher in the tumor invasive edge than the tumor center (intra-TLSs vs. peri-TLSs: $2.19 \pm 2.08$ vs. 4.24 $\pm 2.55, p<0.001$, power $=1.0$ ). Similarly, the infiltrating density of $C D 8+T$ cells and $G r B+T$ cells in the tumor infiltrating edge was significantly higher than in the tumor center (intra-CD8+T vs. peri-CD8+T: $11.40 \pm 6.59$ vs. $13.23 \pm 6.07, p<0.001$, power = 1.0; intra-GrB+T vs. peri-GrB+T: $5.75 \pm 3.83$ vs. $6.88 \pm 4.32, p=0.018$, power $=1.0$; Figure $2 \mathrm{~A}$,
A

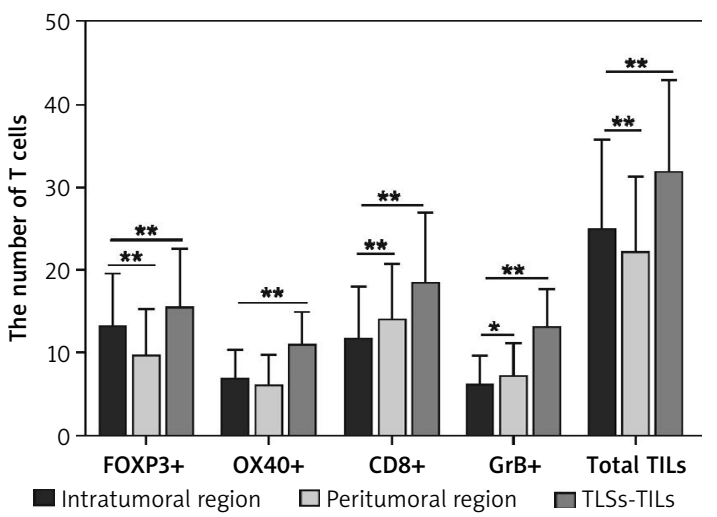

B

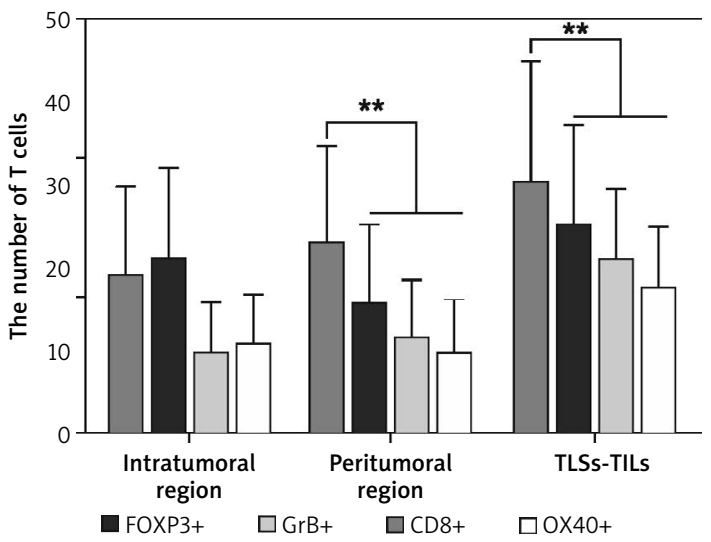

Figure 2. A-TLSs, the total number of TILs and the FOXP3+T cells distributed significantly higher in the tumor center than the tumor invasive edge. While the $\mathrm{CD} 8+$ and $\mathrm{GrB}+\mathrm{T}$ cells in the tumor infiltrating edge were significantly higher than the tumor center. And the four type of TILs within the TLSs is obviously higher than the TILs in intratumoral and peritumoral region $(n=147)$. B - Number of the four subsets of lymphocyte in descending order was $\mathrm{CD} 8+>\mathrm{FOXP} 3+>\mathrm{GrB}+>\mathrm{OX} 40+\mathrm{T}$ cells within the $\mathrm{TLSs}, \mathrm{CD} 8+>\mathrm{FOXP3}+>\mathrm{GrB}+>\mathrm{OX} 40+\mathrm{T}$ cells in the peritumoral region, and the CD8+T cells has a far superior among the TILs in peritumoral region and within the TLSs with statistical significance $(n=147),{ }^{*} p<0.05,{ }^{* *} p<0.01$ 

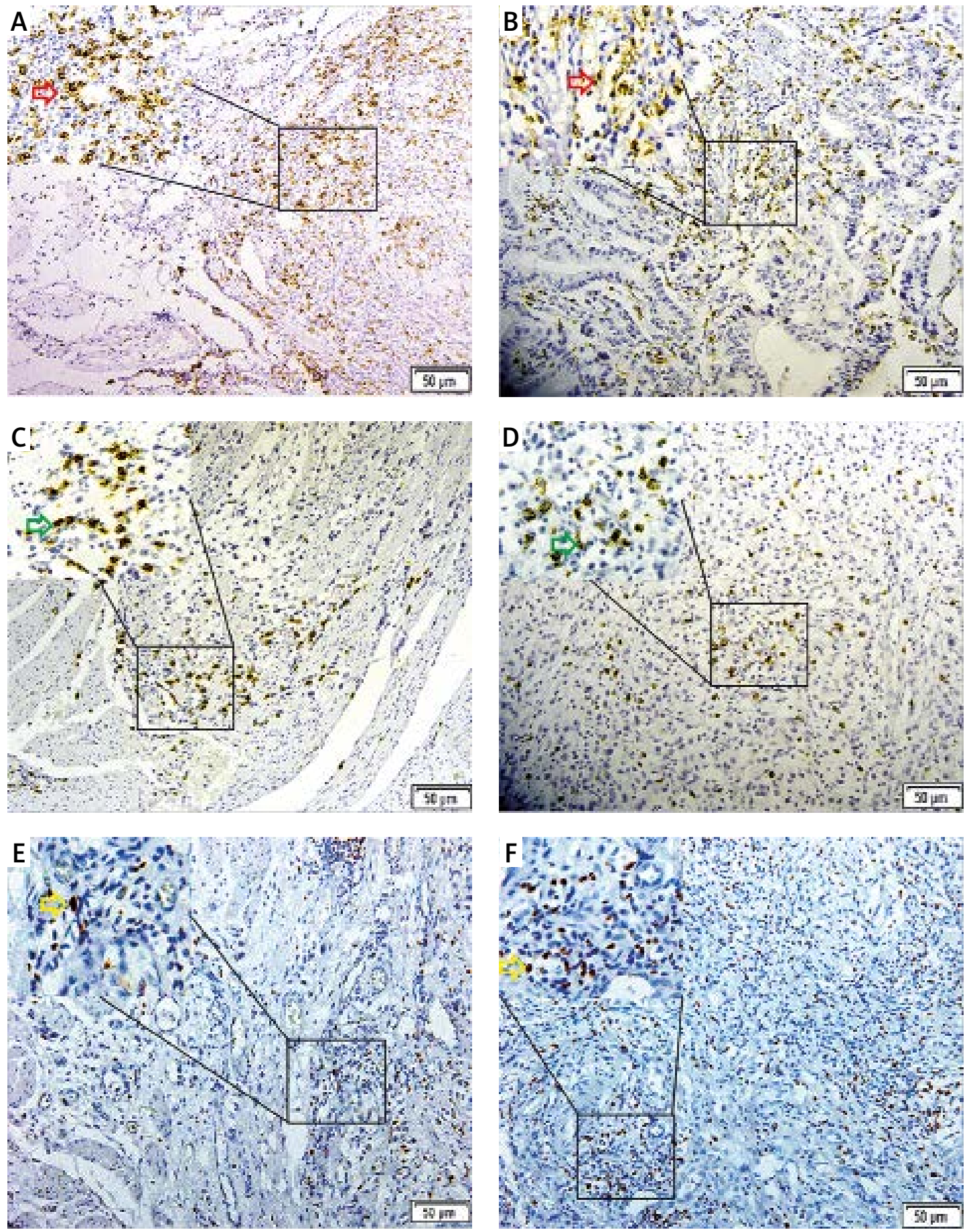

Figure 3. A, C and E - Distribution of CD8+ (red arrow), GrB+ (green arrow) and FOXP3+T cells (yellow arrow) in the peritumoral region. $\mathbf{B}, \mathbf{D}, \mathbf{F}-$ their distribution in the intratumoral region. $\mathbf{G}$ and $\mathbf{H}$ showed the distribution of CD8+ (red arrow) and FOXP3+ (yellow arrow) T cells within the TLSs (rolled up by red and yellow rectangles respectively). The partial enlarged area was in the upper left corner of picture $\mathbf{A}-\mathbf{F}$, and below the picture $\mathbf{G}$ and $\mathbf{H}$

Figure $3 \mathrm{~A}-\mathrm{D})$. The CD8+T cells appeared to form a line of immune cell defense in the tumor-infiltrating edge (Figure $4 \mathrm{~A}, \mathrm{~B}$ ). While the total number of TILs and the FOXP3+T cells was statistically higher in the tumor center than the tumor-infiltrating edge (intra-TILs vs. peri-TILs: $24.73 \pm 10.96$ vs. 21.81 \pm 9.53, $p<0.001$, power = 1.0; intra-FOXP3+T vs.
peri-FOXP3+T $12.88 \pm 6.66$ vs. $9.31 \pm 5.89, p<0.001$ power = 1.0; Figure $2 \mathrm{~A}$, Figure $3 \mathrm{E}, \mathrm{F}$ ), there was no significant difference in the distribution of OX40+T cells in the two regions. The four types of TILs within the TLSs were obviously higher than the TILs in the intratumoral and peritumoral region (Figure $2 \mathrm{~A}$, Figure $3 \mathrm{G}, \mathrm{H}$ ). Additionally, the average 

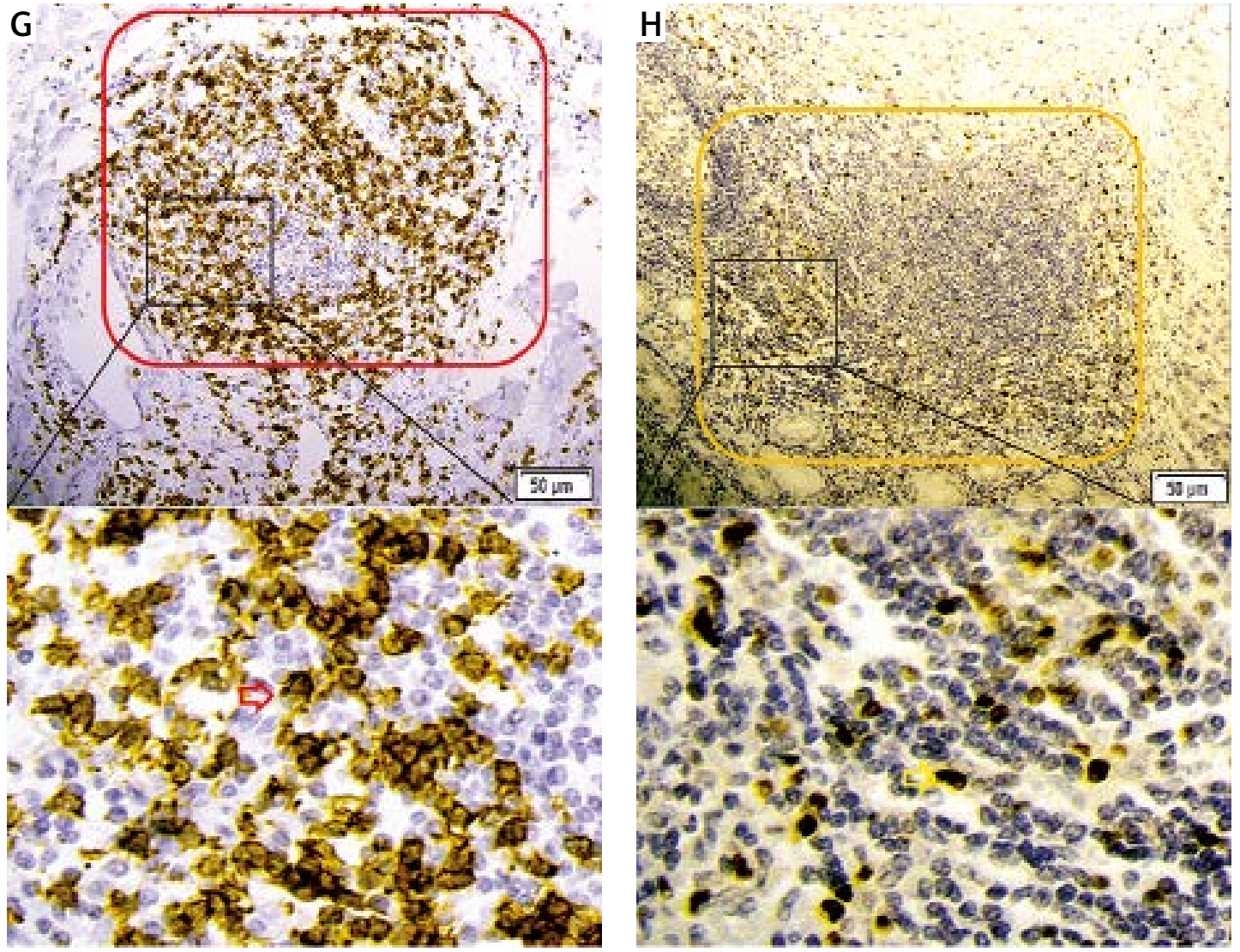

Figure 3. Cont.
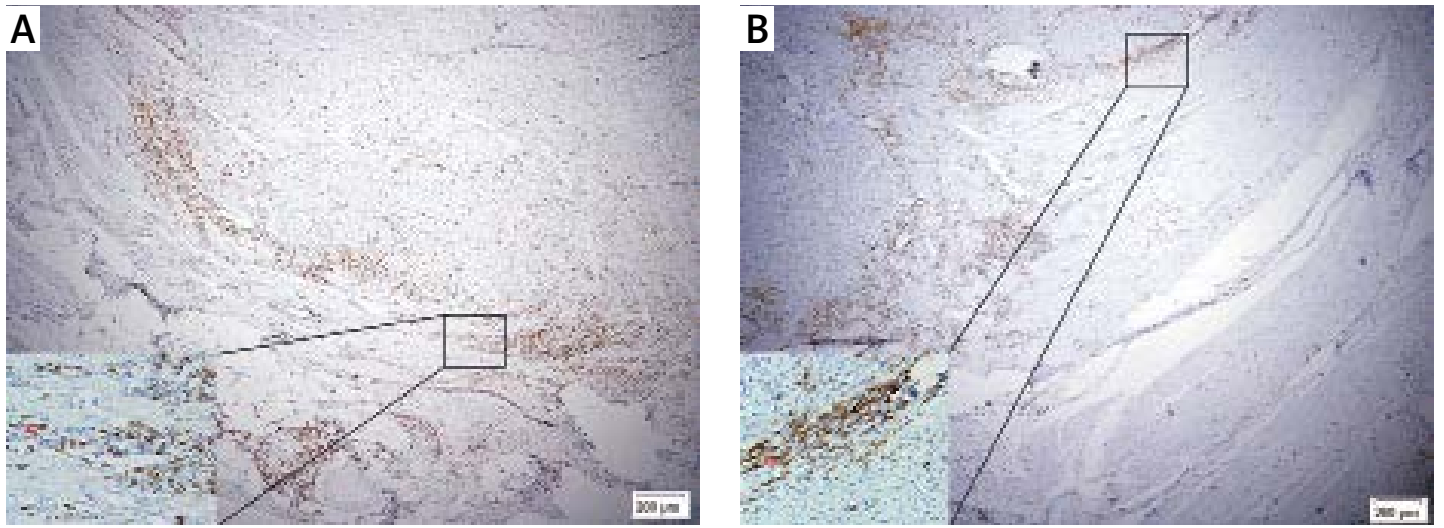

Figure 4. $A$ and $B$ showed the representative image that the CD8+T cells (red arrow) appeared to form a line of immune cell defense in the tumor infiltrating edge. The partial enlarged area was in its lower left corner

number of the four subsets of lymphocytes was $\mathrm{CD} 8+>\mathrm{FOXP3}+>\mathrm{GrB}+>\mathrm{OX} 40+\mathrm{T}$ cells in descending order within the TLSs and $63.27 \%$ of cases were ranked in this order (Figure $2 \mathrm{~B}$ ), indicating that the TLSs were enriched of the cytotoxic T lymphocytes. Moreover, TILs successively arranged in the intratumoral area were $\mathrm{FOXP} 3+>\mathrm{CD} 8+>\mathrm{OX} 40+>\mathrm{GrB}+\mathrm{T}$ cells with $60.54 \%$ of cases in this order, indicating that an immune suppressive microenvironment may exist in the tumor center. In contrast, $C D 8+>$ $\mathrm{FOXP} 3+>\mathrm{GrB}+>\mathrm{OX} 40+\mathrm{T}$ cells were in the peritumor- al region with $61.22 \%$ of cases in this order, and the CD8+T cells are far superior among the TILs in the peritumoral region and within the TLSs with statistical significance (Figure $2 \mathrm{~B}$ ).

There is a positive association between TLSs and TILs in gastric cancer

Correlation analysis showed that the intra-TLSs and peri-TLSs were negatively correlated with each other, though without statistical significance 
Table I. Correlation analysis between TLSs and TILS

\begin{tabular}{|lcccc|}
\hline Subtype of TILs & Location of TLSs and TILs & Correlation coefficient $(r)$ & P-value & Power value \\
\hline FOXP3+T & Intratumor region & 0.077 & 0.357 & 0.153 \\
\cline { 2 - 5 } & Peritumor region & 0.097 & 0.131 & 0.216 \\
\hline OX40+T & Intratumor region & 0.102 & 0.220 & 0.234 \\
\hline CD8+T & Peritumor region & 0.053 & $0.001^{*}$ & 0.050 \\
\cline { 2 - 6 } & Intratumor region & 0.267 & $0.022^{*}$ & 0.058 \\
\hline GrB+T & Peritumor region & 0.189 & $0.044^{*}$ & 0.083 \\
\cline { 2 - 5 } & Intratumor region & 0.166 & 0.061 & 0.114 \\
\hline CD8+FOXP3+T & Peritumor region & 0.155 & $0.043^{*}$ & 0.081 \\
\cline { 2 - 5 } & Intratumor region & 0.168 & $0.047^{*}$ & 0.087 \\
\hline
\end{tabular}

$(r=-0.154, p=0.062$, power $=0.420)$. However, higher amounts of TLSs are always positively correlated with a larger number of TILs either in the location or in the immune type. The total numbers of TILs and CD8+T cells in the intratumoral or peritumoral region and the intra-GrB+T cells were positively related to the TLSs and with statistical significance (Table I).

\section{High density of TLSs and TILs predicts a positive prognostic value of GC}

Higher numbers of peri-TLSs $(p=0.007)$, periCD8+ $(p=0.019)$ and peri-GrB+T cells $(p=0.032)$ were significantly correlated with the favorable survival of GC patients (Figures $5 \mathrm{~A}-\mathrm{C}$ ). Additionally, independently of the location of TLSs and TILS, we found that the survival rate in patients with a higher frequency of TLSs $(p=0.009)$ and CD8+ ( $p=0.033)$ and GrB+T cells ( $p=0.001)$ was significantly better than in those with a lower density of TLSs and TILs (Figure 5 D-F). Also, a higher density of TILs either in the intratumoral $(p=0.025)$ or peritumoral ( $p=0.001$ ) region always predicts better prognosis in GC, regardless of the subtype of TILs (Figure $5 \mathrm{G}, \mathrm{H}$ ).

The Cox proportional hazards model was used to further assess the prognostic value of TLSs and TILs. As shown in Table II, on univariate analysis, the GC patients with lower numbers of TILs ( $\mathrm{F}=4.557, \mathrm{HR}=0.37 ; p=0.008)$ and TLSs $(\mathrm{F}=8.302, \mathrm{HR}=0.51 ; p=0.001)$, older age $(p=0.009)$, and tumors with larger size $(p=0.023)$, positive lymph node metastasis $(p<0.001)$, later stage $(p=0.013)$, and of diffused or mixed type $(p=0.003)$ showed a significantly higher hazard ratio for a poor prognosis. Multivariate analysis $(F=153.434)$ revealed that only positive lymph node metastasis ( $p=0.003)$, frequency of TLSs $(p=0.037)$ and density of TILs $(p=0.019)$ were independent prognostic predictor for GC patients.

\section{Association of TILs and TLSs with clinicopathologic parameters}

Higher frequency of TILs and TLSs was often associated with favorable clinicopathologic parameters. Correlation tests showed that the tumor size was inversely correlated with the TLSs $(r=-0.149$, $p=0.071$, power $=0.439)$ and TILs $(r=-0.286$, $p<0.001$, power $=0.943)$. Additionally, higher density of TILs was significantly associated with smaller tumor size $(p=0.001)$, negative lymph node metastasis $(p=0.009)$, and Lauren classification with an intestinal type $(p=0.002)$. Furthermore, a higher frequency of TLSs was significantly associated with younger age $(p=0.030)$, smaller tumor size $(p=0.037)$, negative lymph node metastasis $(p<0.001)$, and earlier clinical stage $(p<0.001)$ (Table III).

\section{Discussion}

It has been verified that the TLSs could improve local immune responses by recruiting lymphocytes from the blood into tumor tissue [30]. TLSs are structurally characterized by the HEVs that function to recruit lymphocytes into the TME. Studies have shown that the expression of PNAd in HEVs facilitates the migration of lymphocytes from the blood vessel to the tumor tissue [31]. Also, high densities of HEVs correlated with increased naïve, memory, and activated effector memory $T$ cell infiltration and $T$ cell cytotoxicity in human primary solid tumors $[32,33]$. In addition, significantly longer disease-free survival was observed in BCa patients with high-density HEVs than in those with low-density HEVs [34]. Thus, the lymph follicles with positive HEVs in the GC tissue were regarded as the TLSs in this study, and our study showed that a higher frequency of TLSs in GC predicts favorable prognosis no matter where they located.

Similar to the adaptive immune response in SLOs, the dendritic cells recognize, process, and 

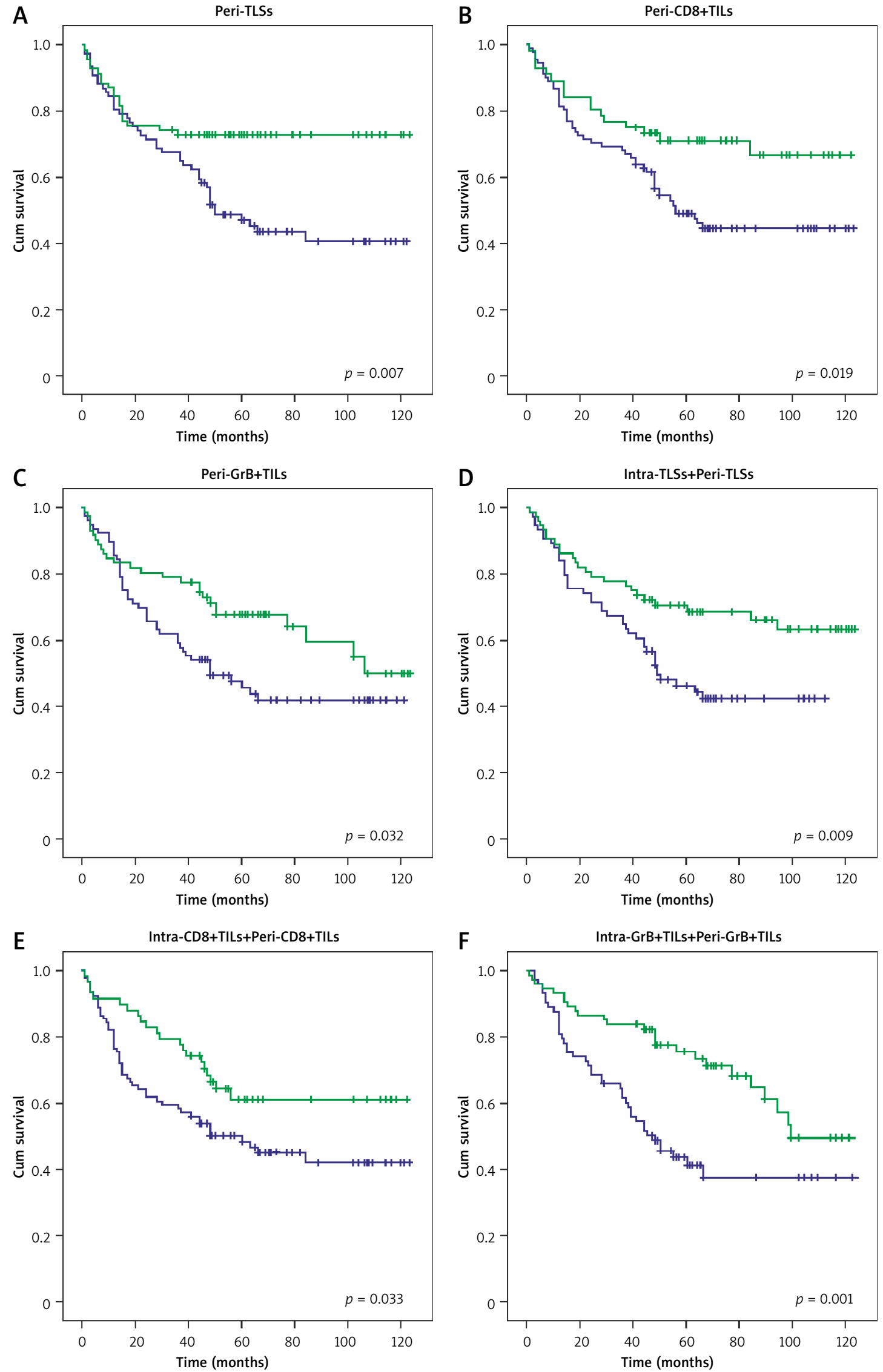

Figure 5. Kaplan-Meier survival analysis with log-rank test. Survival curves of the TLSs and TILs in different region with low and high density 
G

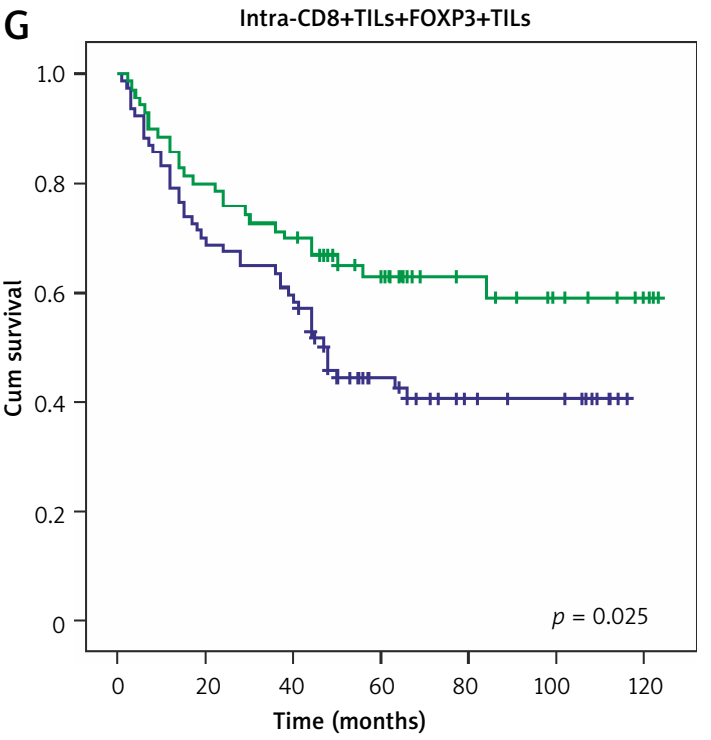

Intra-CD8+TILs+FOXP3+TILs

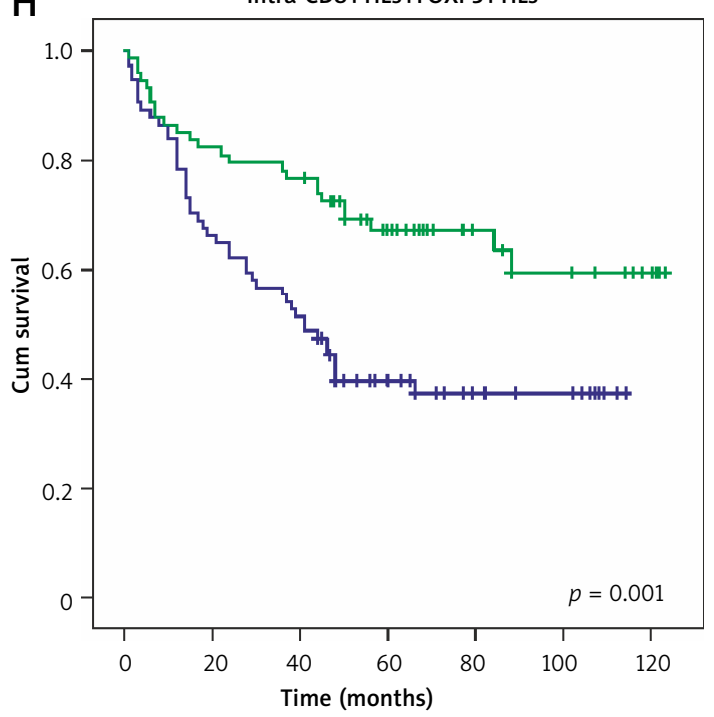

Figure 5. Cont.

Table II. Univariate and multivariate analysis of 10-year overall survival for GC patients

\begin{tabular}{|c|c|c|c|c|c|}
\hline \multirow[t]{2}{*}{ Variables } & \multirow[t]{2}{*}{ Categories } & \multicolumn{2}{|c|}{ Univariate analysis } & \multicolumn{2}{|c|}{ Multivariate analysis } \\
\hline & & $\mathrm{HR}(95 \% \mathrm{Cl})$ & $P$-value & HR $(95 \% \mathrm{Cl})$ & $P$-value \\
\hline Age (years) & $>60($ vs. $\leq 60)$ & $1.25(1.16-9.71)$ & 0.009 & $1.58(0.71-5.21)$ & 0.277 \\
\hline Gender & Male (vs. female) & $1.26(0.78-6.40)$ & 0.540 & & \\
\hline Tumor size $(\mathrm{cm})$ & $>4 \mathrm{~cm}(\mathrm{vs} . \leq 4 \mathrm{~cm})$ & $1.56(1.25-7.75)$ & 0.023 & $2.33(0.93-6.55)$ & 0.226 \\
\hline $\begin{array}{l}\text { Lymph node metastasis } \\
\text { metasisi }\end{array}$ & Positive (vs. negative) & $5.86(3.94-14.96)$ & $<0.001$ & $3.93(1.68-9.61)$ & 0.003 \\
\hline Stage & IIIA-IIIC (vs. IA-IIB) & $2.45(1.87-7.35)$ & 0.013 & $1.86(0.76-6.79)$ & 0.318 \\
\hline Grade & G3-4 (vs. G1-2) & $0.75(0.61-3.91)$ & 0.251 & & \\
\hline Lauren classification & MT+DT (vs. IT) & $1.93(1.73-6.72)$ & 0.003 & $1.66(1.25-5.27)$ & 0.007 \\
\hline TILs & High (vs. low) & $0.37(0.24-0.83)$ & 0.001 & $0.59(0.45-0.74)$ & 0.019 \\
\hline TLSs & High (vs. low) & $0.51(0.46-0.91)$ & 0.008 & $0.64(0.56-0.83)$ & 0.037 \\
\hline
\end{tabular}

present tumor antigens to the T cells, mediating the activation of T cells within the TLSs and attack the tumor cells directly in the TME [5, 25]. Previous studies have verified the different positional role of TILS and TLSs in predicting the prognosis for cancer patients $[28,35,36]$. Additionally, a study has demonstrated that TLSs are important sites for the initiation and/or maintenance of the local and systemic T- and B-cell responses against tumors [7]. Our study indicated that the TLSs were positively correlated with the CD8+TILs and their activated form (GrB+TILs) in GC tissue either in the intratumoral or peritumoral region, but without a significant relation with the CD4+TILs and FOXP3+TILs. It suggested that the TLSs might be the site for the $T$ cell differentiation into the antitumor subtype.

A higher density of $\mathrm{CD} 8+$ and $\mathrm{GrB}+\mathrm{T}$ cells was also verified to be associated with a better outcome for GC patients. Additionally, the number of
CD8+T cells with cytotoxic effects was also significantly higher within the TLSs than FOXP3+T cells with immunosuppressive effects. These results indicated that the formation of TLSs contributes to the differentiation and activation of T cells into an anti-tumor immunophenotype. Previous studies also verified the importance of TLSs in the initiation and/or maintenance of the local and systemic $T$ and $B$ cell responses against tumors [13, 14, 25]. It has been reported that the presence and density of TLS are mainly associated with Th1 and cytotoxic related cellular immune responses in breast, colorectal, lung and pancreatic cancers [25, 27, 37-39]. In addition, TLSs density were also associated with CD20+ B cells, and the size and density of $B$ cell follicles or germinal centers are significantly correlated with good outcome in breast and lung cancer $[13,38]$. Furthermore, in lung cancer and germ cell tumors, the presence of B cell follicles in TLSs promotes the activated mechanisms of ger- 
Table III. Association of TLSs and TILs with clinicopathologic parameters

\begin{tabular}{|c|c|c|c|c|c|c|c|}
\hline \multirow{2}{*}{$\begin{array}{l}\text { Clinicopathologic } \\
\text { parameters }\end{array}$} & \multirow{2}{*}{$\begin{array}{l}\text { No. of cases } \\
\text { (\%) }\end{array}$} & \multicolumn{2}{|c|}{ TIL frequency } & \multirow[t]{2}{*}{$P$-value } & \multicolumn{2}{|c|}{ TLS frequency } & \multirow[t]{2}{*}{$P$-value } \\
\hline & & Low & High & & Low & High & \\
\hline \multicolumn{8}{|l|}{ Age (years) } \\
\hline$\leq 60$ & $79(53.7)$ & 43 & 36 & 0.475 & 30 & 38 & 0.030 \\
\hline$>60$ & $68(46.3)$ & 33 & 35 & & 49 & 30 & \\
\hline \multicolumn{8}{|l|}{ Sex } \\
\hline Female & $31(21.1)$ & 20 & 11 & 0.108 & 18 & 13 & 0.587 \\
\hline Male & $116(78.9)$ & 56 & 60 & & 61 & 55 & \\
\hline \multicolumn{8}{|l|}{ Tumor size $(\mathrm{cm})$} \\
\hline$\leq 4 \mathrm{~cm}$ & $86(58.5)$ & 31 & 55 & 0.001 & 40 & 46 & 0.037 \\
\hline$>4 \mathrm{~cm}$ & $61(41.5)$ & 45 & 16 & & 39 & 22 & \\
\hline \multicolumn{8}{|c|}{ Lymph node involvement } \\
\hline Negative & $62(42.2)$ & 24 & 38 & 0.009 & 14 & 48 & $<0.001$ \\
\hline Positive & $85(57.8)$ & 52 & 33 & & 65 & 20 & \\
\hline \multicolumn{8}{|l|}{ TNM stage } \\
\hline$|A-| \mid B$ & $79(26.5)$ & 45 & 34 & 0.169 & 28 & 51 & $<0.001$ \\
\hline IIIA-IIIC & $68(46.3)$ & 31 & 37 & & 51 & 17 & \\
\hline \multicolumn{8}{|l|}{ Grade } \\
\hline G1-2 & $61(2.0)$ & 29 & 32 & 0.395 & 29 & 32 & 0.204 \\
\hline G3-4 & $86(39.5)$ & 47 & 39 & & 50 & 36 & \\
\hline \multicolumn{8}{|c|}{ Lauren classification } \\
\hline Intestinal type & $86(58.5)$ & 34 & 52 & 0.002 & 49 & 37 & 0.227 \\
\hline Diffuse type & $35(23.8)$ & 25 & 10 & & 20 & 15 & \\
\hline Mixed type & $26(17.7)$ & 17 & 9 & & 10 & 16 & \\
\hline
\end{tabular}

minal centers' somatic hypermutation and class switch recombination, accompanied by generation of plasma cells $[13,40]$. Thus, a high density of TLSs appears to be a good biomarker of the tumor immune microenvironment in which cellular and humoral immune reactions occur. Its favorable prognostic role has also been verified in many different cancer types, including oral squamous cell carcinoma [32], skin Merkel cell carcinoma [41], lung cancer [6,13,14], colorectal cancer [39], pancreatic cancer [26] and breast cancer [37, 38]. The present study showed that the TLS distribution was significantly higher in the tumor invasive edge than the tumor center. Similarly, the $C D 8+T$ cells were also more abundantly distributed in the peritumoral than intratumoral region, and the CD8+T cells were densely distributed within the TLSs. We discovered that the CD8+T cells could form a palisading immune line at the edge of the tumor in many slides (Figures 4 A-B). Studies have reported that tumor cells may harbor some malignant biological behavior at the edge of tumor infiltration, such as poor differentiation, epithelial-mesenchymal transition, stemness differentiation, immune escape and metastatic potential $[42,43]$. Tumor budding, an isolated single cancer cell or a cluster of up to five cells at the invasive front [44] are prone to evolving epithelial-mesenchymal transition, acquiring cancer stem cell biological potential and associated with distant metastasis and poor prognosis for cancer patients $[42,43]$. A higher frequency of TLSs and $\mathrm{CD} 8+\mathrm{T}$ cells in the tumor invasive margin may help to prevent the malignant evolution of cancer cells, and the TLSs can promote the proliferation, differentiation, and activation of CD8+T cells and guide them to migrate to the tumor region $[45,46]$. Therefore, a higher frequency of TLSs is associated with favorable biological indicators and prognosis in this study, which has also been verified by many other studies [6, 7]. Our work also revealed that the TLSs were an independent prognostic factor with a protective effect. However, the prognostic significance of the intratumoral and peritumoral TLSs in this study should be cautiously applied, although many studies also have emphasized the regional importance of immune component in the GC $[35,47]$. Still, there is no exact definition of the peritumoral and intratumoral range of GC, so the prognostic role of different location of TLSs could also differ in different studies. Actually, earlier in 1996, Clemente et al. well documented the evi- 
dence of spatial variations in tumors [48]. It is important to recognize that the microenvironmental components vary significantly in different regions of the tumor tissue[49]. Interestingly, the CD8+T cells and FOXP3+T cells are oppositely distributed in the intratumoral and peritumoral region, and the FOXP3+T cells have lower distribution in the tumor margin and within the TLSs, compared with the CD8+T cells in the present study. A noteworthy study reported that the formation of TLSs and the number of CD8+T cells within TLSs were increased after Treg cells were knocked out in lung cancer mice, and the cancer progression could be inhibited [50]. Another study also documented that Treg cell depletion can enable a self-amplifying loop of T-cell activation, promoting HEV development, T-cell infiltration, and, ultimately, tumor destruction [51]. The specific mechanisms between the Treg cells, CD8+T cells, and TLSs may need to be further investigated.

In conclusion, TLSs can recruit ectopic infiltration of TILs to the GC tissue; simultaneously, the diversity of subtypes of TILs also provides a basis for TLS formation. The TLSs are mostly present in the peritumoral region of the GC tissue, and positively correlated with the infiltration density of $\mathrm{CD} 8+\mathrm{T}$ cells and $\mathrm{GrB}+\mathrm{T}$ cells. GC with higher levels of TLSs and TILs predicts a favorable prognosis. Therefore, the formation of TLS indicates advantageous immune system function, and with a positive anti-tumor effect. TLS might be considered as a novel biomarker to stratify the overall survival risk of untreated GC patients.

\section{Conflict of interest}

The authors declare no conflict of interest.

\section{References}

1. Spill F, Reynolds DS, Kamm RD, Zaman MH. Impact of the physical microenvironment on tumor progression and metastasis. Curr Opin Biotechnol 2016; 40: 41-8.

2. Dunn GP, Old LJ, Schreiber RD. The three Es of cancer immunoediting. Annu Rev Immunol 2004; 22: 329-60.

3. Gajewski TF, Schreiber H, Fu YX. Innate and adaptive immune cells in the tumor microenvironment. Nat Immunol 2013; 14: 1014-22.

4. Jones E, Gallimore A, Ager A. Defining high endothelial venules and tertiary lymphoid structures in cancer. Methods Mol Biol 2018; 1845: 99-118.

5. Pipi E, Nayar S, Gardner DH, Colafrancesco S, Smith C, Barone F. Tertiary lymphoid structures: autoimmunity goes local. Front Immunol 2018; 9: 1952.

6. Dieu-Nosjean MC, Antoine M, Danel C, et al. Long-term survival for patients with non-small-cell lung cancer with intratumoral lymphoid structures. J Clin Oncol 2008; 26: 4410-7.

7. Dieu-Nosjean MC, Giraldo NA, Kaplon H, Germain C, Fridman WH, Sautes-Fridman C. Tertiary lymphoid structures, drivers of the anti-tumor responses in human cancers. Immunol Rev 2016; 271: 260-75.
8. Sautes-Fridman C, Petitprez F, Calderaro J, Fridman WH. Tertiary lymphoid structures in the era of cancer immunotherapy. Nat Rev Cancer 2019; 19: 307-25.

9. Cabrita R, Lauss M, Sanna A, et al. Tertiary lymphoid structures improve immunotherapy and survival in melanoma. Nature 2020; 577: 561-5.

10. Met O, Jensen KM, Chamberlain CA, Donia M, Svane IM. Principles of adoptive $T$ cell therapy in cancer. Semin Immunopathol 2019; 41: 49-58.

11. Molodtsov A, Turk MJ. Tissue resident CD8 memory $\mathrm{T}$ cell responses in cancer and autoimmunity. Front Immunol 2018; 9: 2810.

12. Sautès-Fridman C, Lawand M, Giraldo NA, et al. Tertiary lymphoid structures in cancers: prognostic value, regulation, and manipulation for therapeutic intervention. Front Immunol 2016; 7: 407

13. Germain C, Gnjatic S, Tamzalit F, et al. Presence of B cells in tertiary lymphoid structures is associated with a protective immunity in patients with lung cancer. Am J Respir Crit Care Med 2014; 189: 832-44.

14. De Chaisemartin L, Goc J, Damotte D, et al. Characterization of chemokines and adhesion molecules associated with $\mathrm{T}$ cell presence in tertiary lymphoid structures in human lung cancer. Cancer Res 2011; 71: 6391-9.

15. Dieu-Nosjean MC, Goc J, Giraldo NA, Sautès-Fridman C, Fridman WH. Tertiary lymphoid structures in cancer and beyond. Trends Immunol 2014; 35: 571-80.

16. Kroeger DR, Milne K, Nelson BH. Tumor-infiltrating plasma cells are associated with tertiary lymphoid structures, cytolytic t-cell responses, and superior prognosis in ovarian cancer. Clin Cancer Res 2016; 22: 3005-15.

17. Chen DS, Mellman I. Oncology meets immunology: the cancer-immunity cycle. Immunity 2013; 39: 1-10.

18. Slaney CY, Kershaw MH, Darcy PK. Trafficking of T cells into tumors. Cancer Res 2014; 74: 7168-74.

19. Stanton SE, Disis ML. Clinical significance of tumorinfiltrating lymphocytes in breast cancer. J Immunother Cancer 2016; 4: 59.

20. Zito MF, Ascierto PA, Rossi G, et al. Are tumor-infiltrating lymphocytes protagonists or background actors in patient selection for cancer immunotherapy? Expert Opin Biol Ther 2017; 17: 735-46.

21. Barnes TA, Amir E. HYPE or HOPE: the prognostic value of infiltrating immune cells in cancer. Br J Cancer 2017; 117: 451-60.

22. Reiser J, Banerjee A. Effector, memory, and dysfunctional CD8(+) T cell fates in the antitumor immune response. J Immunol Res 2016; 2016: 8941260.

23. Pages F, Mlecnik B, Marliot F, et al. International validation of the consensus Immunoscore for the classification of colon cancer: a prognostic and accuracy study. Lancet 2018; 391: 2128-39.

24. Liu X, Zhang Z, Zhao G. Recent advances in the study of regulatory T cells in gastric cancer. Int Immunopharmacol 2019; 73: 560-7.

25. Goc J, Germain C, Vo-Bourgais TK, et al. Dendritic cells in tumor-associated tertiary lymphoid structures signal a Th1 cytotoxic immune contexture and license the positive prognostic value of infiltrating CD8+ T cells. Cancer Res 2014; 74: 705-15.

26. Zhang $N$, Wang $D$, Duan $Y$, et al. The special immune microenvironment of tumor budding and its impact on prognosis in gastric adenocarcinoma. Pathol Res Pract 2020; 216: 152926.

27. Hiraoka N, Ino $\mathrm{Y}$, Yamazaki-Itoh R, Kanai $\mathrm{Y}$, Kosuge $\mathrm{T}$, Shimada K. Intratumoral tertiary lymphoid organ is 
a favourable prognosticator in patients with pancreatic cancer. Br J Cancer 2015; 112: 1782-90.

28. Sofopoulos M, Fortis SP, Vaxevanis CK, et al. The prognostic significance of peritumoral tertiary lymphoid structures in breast cancer. Cancer Immunol Immunother 2019; 68: 1733-45.

29. Anderson JA, Dunnill MS. Observations on the estimation of the quantity of emphysema in the lungs by the point-sampling method. Thorax 1965; 20: 462-6.

30. Di Caro G, Marchesi F. Tertiary lymphoid tissue: a gateway for T cells in the tumor microenvironment. Oncoimmunology 2014; 3: e28850.

31. Goc J, Fridman WH, Sautes-Fridman C, Dieu-Nosjean MC. Characteristics of tertiary lymphoid structures in primary cancers. Oncoimmunology 2013; 2: e26836.

32. Wirsing AM, Ervik IK, Seppola M, Uhlin-Hansen L, Steigen SE, Hadler-Olsen E. Presence of high-endothelial venules correlates with a favorable immune microenvironment in oral squamous cell carcinoma. Mod Pathol 2018; 31: 910-22.

33. Sebestyen T, Mohos A, Liszkay G, Somlai B, Gaudi I, Ladanyi A. Correlation with lymphocyte infiltration, but lack of prognostic significance of MECA-79-positive high endothelial venules in primary malignant melanoma. Melanoma Res 2018; 28: 304-10.

34. Martinet L, Garrido I, Filleron T, et al. Human solid tumors contain high endothelial venules: association with T- and B-lymphocyte infiltration and favorable prognosis in breast cancer. Cancer Res 2011; 71: 5678-87.

35. Pak KH, Jo A, Choi HJ, et al. The different role of intratumoral and peritumoral lymphangiogenesis in gastric cancer progression and prognosis. BMC Cancer 2015; 15: 498.

36. Hao J, Li M, Zhang T, et al. Prognostic value of tumorinfiltrating lymphocytes differs depending on lymphocyte subsets in esophageal squamous cell carcinoma: an updated meta-analysis. Front Oncol 2020; 10: 614

37. Martinet L, Filleron T, Le Guellec S, et al. High endothelial venule blood vessels for tumor-infiltrating lymphocytes are associated with lymphotoxin $\beta$-producing dendritic cells in human breast cancer. J Immunol 2013; 191 2001-8.

38. Gu-Trantien C, Loi S, Garaud S, et al. CD4+follicular helper T cell infiltration predicts breast cancer survival. J Clin Invest 2013; 123: 2873-92.

39. Coppola D, Nebozhyn M, Khalil F, et al. Unique ectopic lymph node-like structures present in human primary colorectal carcinoma are identified by immune gene array profiling. Am J Pathol 2011; 179: 37-45.

40. Willis SN, Mallozzi SS, Rodig SJ, et al. The microenvironment of germ cell tumors harbors a prominent antigen-driven humoral response. J Immunol 2009; 182: 3310-7.

41. Behr DS, Peitsch WK, Hametner C, et al. Prognostic value of immune cell infiltration, tertiary lymphoid structures and PD-L1 expression in Merkel cell carcinomas. Int J Clin Exp Pathol 2014; 7: 7610-21.

42. Jun SY, Chung JY, Yoon N, Jung ES, Oh YH, Hong SM. Tumor budding and poorly differentiated clusters in small intestinal adenocarcinoma. Cancers (Basel) 2020; 12: 2199.

43. Li H, Xu F, Li S, Zhong A, Meng X, Lai M. The tumor microenvironment: An irreplaceable element of tumor budding and epithelial-mesenchymal transition-mediated cancer metastasis. Cell Adh Migr 2016; 10: 434-46.

44. Prall F. Tumour budding in colorectal carcinoma. Histopathology 2007; 50: 151-62.
45. Teillaud JL, Dieu-Nosjean MC. Tertiary lymphoid struc tures: an anti-tumor school for adaptive immune cells and an antibody factory to fight cancer? Front Immunol 2017; 8: 830 .

46. Bourges D, Zhan Y, Brady JL, et al. Targeting the gut vascular endothelium induces gut effector CD8 T cell responses via cross-presentation by dendritic cells. J Immunol 2007; 179: 5678-85.

47. Wang XL, Fang JP, Tang RY, Chen XM. Different significance between intratumoral and peritumoral lymphatic vessel density in gastric cancer: a retrospective study of 123 cases. BMC Cancer 2010; 10: 299.

48. Clemente CG, Mihm MC Jr, Bufalino R, Zurrida S, Collini P, Cascinelli N. Prognostic value of tumor infiltrating lymphocytes in the vertical growth phase of primary cutaneous melanoma. Cancer 1996; 77: 1303-10.

49. Yuan Y. Spatial heterogeneity in the tumor microenvironment. Cold Spring Harb Perspect Med 2016; 6: a026583.

50. Joshi NS, Akama-Garren EH, Lu Y, et al. Regulatory T cells in tumor-associated tertiary lymphoid structures suppress anti-tumor T cell responses. Immunity 2015; 43 579-90.

51. Colbeck EJ, Jones E, Hindley JP, et al. Treg depletion licenses $\mathrm{T}$ cell-driven HEV neogenesis and promotes tumor destruction. Cancer Immunol Res 2017; 5: 1005-15. 